\title{
Monitoring Variability of the Sky
}

\author{
Bohdan Paczyński \\ Princeton University, Princeton, NJ 08544-1001, USA
}

\begin{abstract}
Variability in the sky has been known for centuries, even millennia, but our knowledge of it is very incomplete even at the bright end. Current technology makes it possible to built small, robotic optical instruments, to record images and to process data in real time, and to archive them on-line, all at a low cost. In addition to obtaining complete catalogs of all kinds of variable objects, spectacular discoveries can be made, like the optical flash associated with GRB 990123 and a planetary transit in front of HD 209458. While prototypes of parts of such robotic instruments have been in operation for several years, it is not possible to purchase a complete system at this time. I expect (hope) that complete systems will become available 'off the shelf' in the near future, as monitoring bright sky for variability has a great scientific, educational and public outreach potential.
\end{abstract}

\section{Introduction}

During the last decade several billion dollars have been spent worldwide to build 6.5-10 meter class telescopes, and there are about 15 of those giants in operation or under construction. With ever larger apertures and ever more sophisticated detectors it is possible to study the universe not only in ever greater detail but also to make entirely new and very important discoveries. However, in this very expensive race to reach the faintest objects, with the highest angular and spectral resolution and over the widest spectral range, a broad area of research has been largely neglected: the monitoring of optical sky for variability.

The all sky monitors were known in X-ray and gamma-ray domains for many decades. The two examples are: Compton GRO (exists no more)

and Rossi XTE (still in operation)

$$
\text { http://cossc.gsfc.nasa.gov/cossc/ }
$$

$$
\text { http://heasarc.gsfc.nasa.gov/docs/xte/xte_1st.html }
$$

capable of monitoring gamma-ray and X-ray variability on time scales from milliseconds to years over the whole sky. And there is BACODINE system with the GCN electronic circulars

$$
\text { http://gcn.gsfc.nasa.gov/gcn/gcn_main.html }
$$

which provides worldwide distribution of 'what is new' in the X-ray and gammaray sky. But there is no such rapid discovery and distribution system in the optical domain. True, BACODINE/GCN is used by optical and radio observers to report their follow-up work triggered by X-ray and gamma-ray events. Also, gravitational microlensing, supernovae and asteroid searches provide optical 
alerts for very limited areas in the sky. Yet, there is no optical system capable of recognizing that something new and unexpected is happening anywhere in the sky, and to instantly verify the discovery. The only all sky optical monitoring is done by amateurs using the naked eye, and therefore it is limited to $4 \mathrm{mag}$, or so, with the verification and follow-up possible on a time scale of hours or days, but not seconds, as it is the case with the BACODINE/GCN.

Professional astronomers do not appreciate how under-explored is the sky variability, even at the bright domain. The ASAS (Pojmański 2000) and ROTSE (Akerlof et al. 2000) projects demonstrated that by using a $10 \mathrm{~cm}$ aperture it is possible to increase the number of known variables brighter than $13 \mathrm{mag}$ by a factor of 10. ROTSE (Akerlof et al. 1999) and STARE (Charbonneau et al. 2000) demonstrated that it is possible to make very important discoveries with such small apertures: the optical flash from a redshift $\mathrm{z}=1.6$, and the planetary transit in front of a star. But note: these two spectacular discoveries were made in a follow-up mode, with the target area in the sky, and the target star, selected with expensive space instruments (BATSE, BeppoSAX) or a large optical telescope. The existing hardware: small, inexpensive robotic instruments, can detect optical flashes and planetary transits, but the software required to make independent discoveries does not exist, and there appears to be little will to develop it, though check McGruder (2001) and STARE:

http://www.hao.ucar.edu/public/research/stare/stare.html

In this presentation I am making a case for small instruments. There is a lot of science to be done, but to be efficient, and therefore effective, the small instruments must be fully robotic. It is a great pleasure to develop and operate such instruments: no need to struggle with TACs (time allocation committees), no need to write observing proposals. A large team with all the managerial and funding problems is not necessary, as a full system can be developed with very modest funds by a competent individual or a small group, as demonstrated by ASAS (Pojmański 2001):

http://archive.princeton.edu/ ${ }^{\sim}$ asas/

Still, it is not trivial to develop fully robotic hardware, and robust software is the main bottleneck.

\section{Today's Systems}

There are several projects which use small robotic instruments to image almost all sky every clear night, or every few nights. Almost all of them are focused on specific targets, usually searching for optical flashes associated with gamma-ray bursts, and archiving data with no serious attempt to analyze it. The volume is huge, in some cases several terabytes, so data handling is not easy, and data analysis seems beyond the capability or interest of large teams involved, like ROTSE, LOTIS, TAROT, STARE. Links to their Web sites may be found at http://www.astro.princeton.edu/faculty/bp.html http://alpha.uni-sw.gwdg.de/ ${ }^{\sim}$ hessman/MONET/

There are many projects taking data, but as far as I know only one of them monitors everything that varies within its field of view: the All Sky Automated Survey. Interestingly, ASAS is a single person undertaking (Pojmański 2000, 2001). Unfortunately, even ASAS is still processing data off-line. Another small 
group, OGLE (Udalski et al. 1997):

$$
\text { http://bulge.princeton.edu/ ogle/ }
$$

is processing all data almost in real time, but its Early Warning System (EWS) alerts on microlensing events only, on a time scale of a day or so, and it monitors less than 0.001 of the sky. I expect that within a year or so ASAS's software will be like OGLE's, perhaps even faster, with the alert time scale of minutes, and near real time verification of anomalous photometric and/or astrometric variation of any type. Perhaps some other team will reach this capability ahead of ASAS. It would be great if this became a common mode of operation.

Unfortunately, the present small robotic instruments are little more than prototypes. It is not possible to order and purchase a complete system, or just a complete hardware, for a known price. The only exception is CONCAM, a very compact camera (cf. Nemiroff et al. 2000, Pereira et al. 2000, Perez-Ramirez et al. 2000):

\section{http://concam.net/}

capable of imaging all sky every few minutes using a CCD detector with a relatively small number of pixels, controlled by a lap-top computer. Unfortunately, at this time no photometric/astrometric pipeline software exists for CONCAM. In general, no complete and portable software package is available for any small instrument. This means that most of the data are just archived, and never fully processed. I suppose this is not unusual for a field which is in its early stages of development, with relatively few people involved, and even fewer people convinced that it is scientifically useful to have open-minded rather than narrowly focused observing projects.

\section{Scientific and Educational Goals}

The list of known types of variable objects is long. It includes eclipsing, pulsating, and exploding stars, active galactic nuclei (AGNs), asteroids, comets, and a large diversity of optical flares or flashes. Scientific goals are very diverse. Complete catalogs of variable stars are needed for studies of galactic structure and stellar evolution. Calibration of various distance indicators can be done with the nearest, and therefore apparently the brightest objects. AGNs are still poorly understood, and they vary on all time scales longer than an hour or so. Comets and asteroids are important for studies of the solar system, while 'killer asteroids' have a great potential for entertainment, as most of them are not deadly at all, just spectacular. Finally, with so many big telescopes in operation and under construction it is useful to have a variety of targets of opportunity detected in real time (cf. Paczyński 1997, 2000, Nemiroff \& Rafert 1999, and references therein).

What makes small instruments scientifically interesting is the very high data rate which can be generated and processed at low cost, provided suitable software is available. With the gradual decrease of detector prices it is possible to have a large number of pixels. Computer power is increasing and its cost is falling all the time. The operating expenses of OGLE hardware translate to over 100,000 photometric measurements per $\$ 1$. By the time these proceedings are published the cost of 1 terabyte of IDE disk will be about $\$ 2,000$, making it possible to have huge data sets on-line. So, it makes sense to use a 'vacuum 
cleaner' approach and to process all CCD frames and convert 'pixel data' into 'catalog data', which can be analyzed by a much broader range of astronomers, even amateurs. The diversity of data types is small, as all variables are point sources, but the diversity of phenomena is large, making the database interesting for a variety of scientific as well as educational projects.

With an avalanche of data a small team like OGLE or ASAS cannot possibly analyze it all. A question comes up: should the data be kept in a closet for future analysis, or should it be made public domain so other astronomers can do science with it now? My view, as well as the view of the OGLE and ASAS teams, is that the latter solution is preferable; with the data rate increasing exponentially there will never be time to analyze it all internally. An example of this policy is a recent publication by the OGLE team of almost $1,000,000$ photometric measurements for almost 4,000 Cepheids in the LMC and SMC (Udalski et at. 1999a,b). The data posted on the web was analyzed by Dr. D. S. Graff of the Ohio State University, who noticed a small but clear systematic error reaching several hundreds of a magnitude near the edges of OGLE images. The error was verified and the electronic archive was revised on April 1, 2000 . Subsequently, the data was successfully used to study the geometry of LMC and SMC (Groenewegen 2000). While this work was being done elsewhere the OGLE team had time to work on other projects, and Dr. A. Udalski had time to work on a new, large CCD camera for OGLE.

Some focused projects may have very diverse applications. Let me give two examples.

The very successful Katzman Automatic Imaging Telescope (Filippenko 2001) discovers dozens of relatively nearby supernovae every year. It is not an all sky system, but it monitors several thousand galaxies. It would be great if the Katzman system could be copied, and the detection rate of supernovae increased to $\sim 1,000$ per year. Who needs so many events? One of the most outstanding unsolved problems in modern astrophysics is a relation between supernovae and gamma-ray bursts (GRBs). It is likely that GRBs are strongly beamed (cf. Frail et al. 2001, and references therein). If the true GRB rate is $\sim 1,000$ times higher than the observed rate, then up to $1 \%$ of all supernovae may generate a gamma-ray burst which in most cases is not beamed at us. However, its afterglow may become detectable as a bipolar radio-supernova remnant several years after the explosion. Some supernovae are followed by a strong radio signal which can be detected with the VLA. The few sources which are strong enough could be followed-up with the VLBA with a sub-milli-arcsecond resolution. The explosions related to GRBs which are not beamed at us could be recognized by their bipolar structure and relativistic expansion. We need as many nearby supernovae as possible to have a chance to discover those hypothetical bipolar radio remnants (Paczyński 2001)

A search for near Earth asteroids (NEAs) is aimed at the discovery of all (or at least most) 'killer asteroids'. These are objects which could, upon impact, cause global catastrophe. The minimum diameter is estimated to be $\sim 1 \mathrm{~km}$. Hundreds of such asteroids were already discovered by many projects, like

LINEAR, http://www.ll.mit.edu/LINEAR/

LONEOS, http://asteroid.lowell.edu/asteroid/loneos/loneos_disc.html NEAT, http://neat.jpl.nasa.gov/ 
SPACEWATCH, http://www.lpl.Arizona.edu/spacewatch/

An up to date information may be found at

MPC, http://cfa-www.harvard.edu/cfa/ps/mpc.html

and a recent review was written by Ceplecha et al. (1998). The searches discovered also a large number of smaller objects, down to several meter diameter. It turns out that about once a month an asteroid 12 meters across, with a mass of $\sim 1,000$ tons collides with Earth and releases in the upper atmosphere $\sim 10$ kilotons of TNT equivalent. Ten times more energetic events happen once a year. These are spectacular fireballs with strong acoustic effects, but no harm is done at the ground level. About once a century a Tunguska-like event releases up to 10 megaton in an explosion which is locally devastating.

The searches for near Earth asteroids are done with 1 meter class telescopes, which implies that a major part of the sky is covered once every week or so. This is frequent enough to discover a broad range of asteroid sizes, and to make statistical estimates of the probability of impacts, but not frequent enough to recognize those few which are about to collide with Earth. Naturally, the huge data archive contains information about many types of objects with variable brightness, but they are ignored unless they also change their position. This is a huge untapped treasure with the information of general variability of thousands, perhaps millions of stars and AGNs. In the next section I shall discuss modest extensions of the current asteroid searches which could provide alerts about impending impacts as well as alerts for any unusual variability in the sky.

Educational opportunities offered by small telescopes are very well described elsewhere in these proceedings (Hessman 2001). Unfortunately, neither OGLE nor ASAS has an active educational program so far.

\section{Prospects for the Future}

With all the current, very diverse activity one goal has not been achieved so far: we do not know what is happening in the sky in real time, even at the bright end. This is a huge gap in astronomical research, which can be filled only with small, wide angle instruments. The goal is to monitor all sky at the shortest possible time intervals down to whatever magnitude limit is technically and financially feasible, to process the data as soon as it is acquired, to send alerts, and to archive the results in public domain, so that broad scientific analysis can be accessible to many users, who could be called virtual observers.

There are several obvious steps to be made for this idea to become viable. First, a complete system with fully automated hardware and software pipeline and real time alert system should become operational - none exists at this time. Next, hardware should be made easy to duplicate, to allow for a relatively simple expansion to various sites and various groups. The availability and the cost of all the components should be known, and it should be low. I expect software to be public domain and free. Once the systems spread it will be necessary to find a way to coordinate the data flow, the diversity of alerts and the ever growing on-line archive. I think there will be many problems which are impossible to predict and we should be open-minded and flexible. It is very likely that several distinctly different systems will be developed, with a broad range of costs, data rates, depths and the scope of surveys. 
Notice that optical variability may have a time scale as short as a microsecond, as long as the age of the universe, and anything in between. It is obviously impossible to cover the whole sky every second to $24 \mathrm{mag}$. But it is relatively easy to cover it down to 10 mag every minute, and this may be a good start, or a good followup on the CONCAM. The search space is multi dimensional: how large area in the sky is monitored, how often, how deep, in which filters, with what accuracy? All past and current searches operate in some area of this parameter space, and there is no way to know where the most spectacular and unexpected discoveries are to be made. Consider an example: for over a century enigmatic super-flares were observed on normal main sequence, single, slowly rotating stars of F8 - G8 type (Schaefer 1989, Schaefer et al. 2000). However, an instant follow-up was never done and their nature, or even their reality is not known. Perhaps they are not actually on the stars, but on companion planets (Rubenstein 2001)?

A serious discussion is under way to define science to be done with the LSST (Large Synoptic Survey Telescope = Dark Matter Telescope, Tyson et al. 2000). This is a project to build a telescope with a fast 8.4 meter mirror, a field of view 3 degrees across, and 1.4 Giga pixels. If built, it will be able to image all sky in just a few nights, reaching $24 \mathrm{mag}$, and saturating at 15 mag. As powerful as it will be, LSST will not be likely to discover an optical flash like the one associated with GRB 990123, as at any given time LSST will image only $\sim 0.0001$ of the sky. Of course, LSST will detect a large number of very interesting faint transients. But note: it is much easier to follow-up an optical flash which peaks at $10 \mathrm{mag}$ than one that peaks at $24 \mathrm{mag}$, yet the bright sky variability on a time scale of seconds or minutes is not explored at all.

A detection of small asteroids about to collide with Earth should be possible several hours or even days prior to their impact. The alerts would be useful not only scientifically, but they also would be great for public outreach and entertainment if the time and location of the next explosive fireball in the upper atmosphere could be predicted. Such alerts are not possible now, but several near flybys were reported. A few years ago a graduate student in Tucson, Timothy Spahr, discovered a 300 meter diameter asteroid $1996 \mathrm{JA1}$, passing within 450,000 kilometers of Earth (Spahr 1996). It reached 11 mag at the closest approach:

\section{http://cfa-www.harvard.edu/cfa/ps/mpec/J96/J96K06.html}

A typical relative velocity of an approaching asteroid is $\sim 14 \mathrm{~km} / \mathrm{s}$, which implies $\sim 8$ hour time to reach Earth from the Moon distance. A rock with a 30 meter diameter appears as a $\sim 15 \mathrm{mag}$ object at $400,000 \mathrm{~km}$. A 12 meter rock would be $\sim 14$ mag two hours prior to its impact. Such objects collide with Earth once per year and once per month, respectively. Obviously, detecting them is not easy, but it is not outside the range of current technology. If recognized as heading our way the follow-up observations and the determination of their trajectory would have to be done very quickly, and presumably automatically, in order to make a prediction of the time and the location of their impact. The publicity would be justified, even for near misses, i.e. near Earth flybys. A major asteroid or comet on a Tunguska scale, with a $\sim 100$ meter diameter, might be detected several days prior to its impact, providing enough time to evacuate the 'ground zero'. 
There is no obvious limit to the expansion of all sky monitoring. Gradual reduction of detector and computer costs will make it possible to cover all sky every night, every hour, every minute, to ever lower flux limits. Modest scientific returns are to be expected even for a project reaching 14 mag every night (like ROTSE) or 10 mag every minute (a bit better than CONCAM) provided the data analysis is automated and real time alerts of any unusual variability are implemented.

Acknowledgments. It is a pleasure to acknowledge the support by NSF grants AST-9819787 and AST-9820314.

\section{References}

Akerlof, C. et al. (ROTSE collaboration) 1999, Nature, 398, 400

Akerlof, C. et al. (ROTSE collaboration) 2000, AJ, 119, 1901

Ceplecha, Z. et al. 1999, Space Sci. Rev., 84, 327

Charbonneau, D. et al. 2000, ApJ, 529, L45

Filippenko, A. 2001, these proceedings

Frail, D. A. et al. 2001, astro-ph/0102282

Groenewegen, M. A. T. 2000, astro-ph/0010298

Hessman, F. V. 2001, these proceedings

McGruder, C. 2001, these proceedings

Nemiroff, R. J. \& Rafert, J. B. 1999, PASP, 111, 886

Nemiroff, R. J. et al. 2000, AAS Meeting 197, 120.04

Paczyński B. (1997) Proc. 12th IAP Colloquium: 'Variable Stars and the Astrophysical Returns of Microlensing Searches', Paris (Ed. R. Ferlet), p. 357

Paczyński, B. 2000, PASP, 112, 1281

Paczyński, B. 2001, AcA, 51, 1

Perez-Ramirez, D. et al. 2000, AAS Meeting 197, 115.08

Pereira, W. E. et al. 2000, AAS Meeting 197, 115.10

Pojmański, G. (ASAS) (2000) AcA, 50, 177

Pojmański, G. (ASAS) (2001) these proceedings

Rubenstein, E. P. 2001, astro-ph/0101573

Schaefer, B. E. 1989, ApJ, 337, 927

Schaefer, B. E., King, J. R. \& Deliyannis, C. P. 2000, ApJ, 529, 1026

Spahr, T. 1996, IAUC No. 6402

Tyson, J. A., Wittman, D. \& Angel, J. R. P. 2000, astro-ph/0005381

Udalski, A., Kubiak, M. \& Szymański, M. 1997, AcA, 47, 319

Udalski, A. et al. 1999a, AcA, 49, 223

Udalski, A. et al. 1999b, AcA, 49, 437 

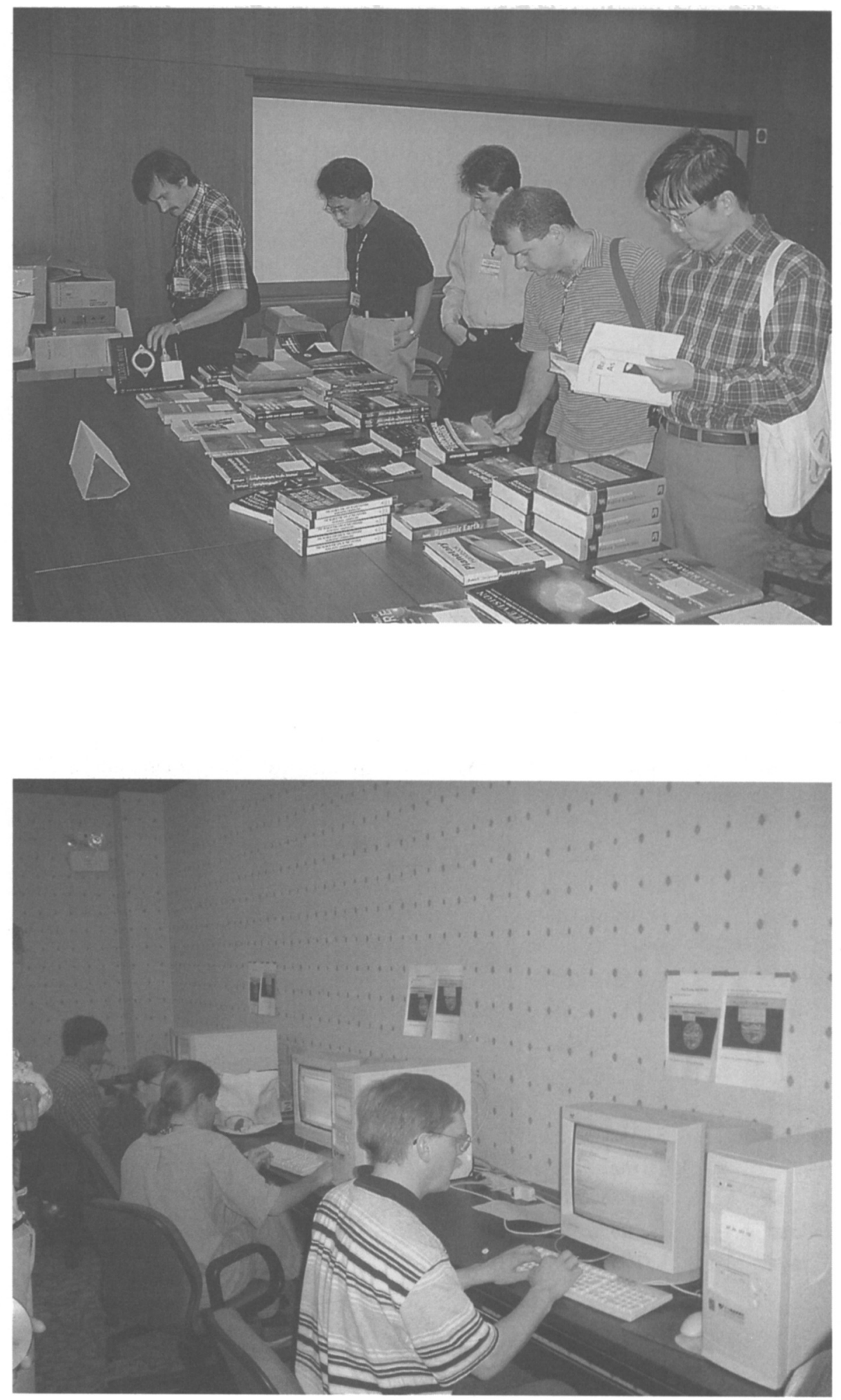